\title{
VALEUR ALIMENTAIRE DES LEVURES \\ II. - INFLUENCE DU MODE DE SÉCHAGE DE QUELQUES LEVURES DE DISTILLERIE
}

\author{
PAR \\ R. FEVRIER \\ Station de Recherches sur 1'Êlevage, Jouy-en-Josas (S.-et-O.).
}

Ce travail, réalisé à Jouy-en-Josas pendant 1'été I953, constitue la suite de celui qui a été publié en 1952 (Ann. Zoot., no 4 , p. I-Io). Il porte sur deux nouvelles séries de levures de distillerie dont nous avons voulu comparer la "valeur alimentaire " sur la croissance du porc.

Ces levures ont été obtenues dans les conditions suivantes:

Série $I$ :

- levure $Y_{1}$ : levure de betterave séchée à haute température,

- levure $\mathrm{Y}_{2}$ : levure de betterave séchée à haute température et séparée après la colonne à distiller,

- levure $\mathrm{Y}_{3}$ : torula de vinasse de mélasse séchée à haute température.

Ces échantillons provenaient de 3 usines différentes (campagne r952I953).

Série II:

- levure $Z_{\mathbf{4}}$ : levure de mélasse séchée à basse température,

- levure $\mathrm{Y}_{4}$ : levure de mélasse séchée à haute température.

Ces deux échantillons provenaient de la même usine normalement équipée pour travailler à basse température, mais qui, pour la campagne I95I-52, avait séché une certaine quantité de levure à haute température à titre d'essai.

Les techniques expérimentales utilisées sont, dans l'ensemble, les mêmes que celles décrites en I952, avec toutefois les modifications suivantes:

a) Le taux de levure a été abaissé à 8-9\%, le pourcentage d'azote provenant des levures étant le même dans toutes les rations. De cette façon, les inconvénients éventuels, sur le plan expérimental, d'une ingestion massive de levure étaient évités.

b) Pour obtenir le taux d'azote requis par les normes, nous avons utilisé un tourteau d'arachide. Ses protéines, de valeur biologique 
moyenne, ne pouvaient masquer que faiblement les différences de valeur existant entre les protéines des levures.

c) Nous avons constitué les lots de porcelets de la façon suivante : dans chaque portée dont nous disposions, plusieurs groupes de 2 ou 3 sujets semblables furent constitués : à l'intérieur de chaque groupe, chacun des animaux fut, par tirage au sort, affecté à un traitement. L'opération fut répétée un certain nombre de fois : 9 fois pour la série $I, 8$ fois pour la série II. La comparaison des croissances, consommations, etc... put donc être effectuée entre les animaux de chaque groupe et répétée 8 ou 9 fois, selon les séries.

d) Pour que les comparaisons soient effectuées dans le même intervalle de croissance pour tous les animaux, chaque sujet a été observé à partir du moment où il pesait 20 à $25 \mathrm{~kg}$ (les pesées étaient hebdomadaires) jusqu'à ce qu'il atteigne 75 à $80 \mathrm{~kg}$ pour la série I et $5^{2-58} \mathrm{~kg}$ pour la série II.

Les régimes employés avaient la composition suivante:

\begin{tabular}{|c|c|c|c|c|}
\hline & $Y_{1}$ & $Y_{2}$ & $Y_{3}$ & $Z_{4}$ \\
\hline 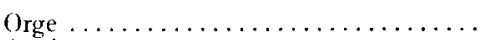 & 50 & 50 & 50 & 48 \\
\hline Avoine $\ldots \ldots \ldots \ldots \ldots$ & 28 & 28 & 29 & 29 \\
\hline Tourteau d'arachide .... & 5 & 5 & 5 & 5 \\
\hline Farine de luzerne .. & 5 & 5 & 5 & 5 \\
\hline Mélange minéral.. & 3 & 3 & 3 & 3 \\
\hline Ievure ........ & 9 & 9 & 8 & 10 \\
\hline
\end{tabular}

I, 'analyse des levures donna les résultats suivants:

\begin{tabular}{|c|c|c|c|c|c|}
\hline & $Y_{1}$ & $Y_{2}$ & $\mathrm{Y}_{3}$ & $Z_{4}$ & $\mathrm{Y}_{4}$ \\
\hline Matière sèche p. 1000 & $895, \circ$ & 860,3 & 946,8 & 863,4 & 857,2 \\
\hline atiè & 75,5 & 34,9 & 107,8 & & 64,8 \\
\hline atières cellulosiques p. I 000 . & 57,7 & 75,0 & 40,8 & 66,7 & 54,9 \\
\hline Matières grasses p. I $000 . .$. & 28,2 & 33,5 & $5 \mathrm{I}, 7$ & 19,8 & 22,8 \\
\hline Matières azotées p. I $000 \ldots \ldots$. & $445, \mathrm{I}$ & 442,4 & $5 \mathrm{I} 7, \mathrm{I}$ & 416,0 & 392,0 \\
\hline Vitamine $\mathrm{B}_{2}{ }^{(1)}$ (mg par $\left.\mathrm{kg} \mathrm{sec}\right)$. & 43,0 & 6,0 & 62,7 & 26,8 & 27,3 \\
\hline Vitamine $\mathrm{PP}(\mathrm{mg}$ par $\mathrm{kg} \mathrm{sec}) .$. & 164,0 & 8,0 & 442,0 & 597 & $54^{2}$ \\
\hline
\end{tabular}

Les résultats observés sur les animaux sont présentés dans les deux tableaux suivants :

Série I:

Nombre d'animaux

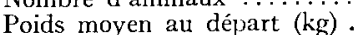

Poids moyen au départ $(\mathrm{kg}) \ldots \ldots \ldots \ldots \ldots$

Poids moyen à la fin $(\mathrm{kg}) \ldots \ldots \ldots \ldots \ldots \ldots$

Gain moyen quotidien (g./jour) ...........

Consommation moyenne quotidienne $(\mathrm{kg}) \ldots$...

Indice moyen de consommation ............

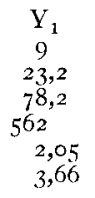

Série 11 :

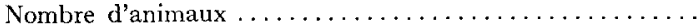

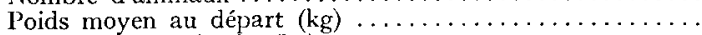

Poids moyen à la fin $(\mathrm{kg}) \ldots \ldots \ldots \ldots \ldots \ldots \ldots \ldots \ldots \ldots$

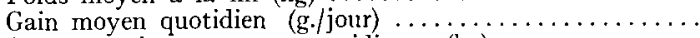

Consommation moyenne quotidienne $(\mathrm{kg}) \ldots \ldots \ldots \ldots \ldots$

Indice moyen de consommation $\ldots \ldots \ldots \ldots \ldots \ldots \ldots \ldots$

$\begin{array}{cc}\mathrm{Y}_{2} & \mathrm{Y}_{3} \\ 9 & 9 \\ 24,5 & 23,3 \\ 77,7 & 78,0 \\ 5 \mathrm{I} 8 & 559 \\ \mathrm{I}, 94 & 2,07 \\ 3,76 & 3,72\end{array}$

$\begin{array}{cc}Z_{4} & \mathrm{Y}_{4} \\ & \frac{8}{8} \\ 23,4 & 23,4 \\ 55,4 & 56,2 \\ 529 & 594 \\ \mathrm{I}, 95 & \mathrm{I}, 80 \\ 3,68 & 3,04\end{array}$

(1) Nous remercions M. L. Chevillard, Directeur du Laboratoire de Biochimie des Vitamines à l'École des Hautes-Études, d'avoir bien voulu doser diverses vitamines dans les levures étudiées. 


\section{Examen des résultats:}

Notons tout d'abord que ces résultats sont du même ordre de grandeur que ceux obtenus couramment avec une alimentation équilibrée.

L'analyse statistique de ces résultats montre que, dans la série II, les différences sont significatives : au niveau 0,05 pour la vitesse de croissance - au niveau o,or pour la consommation et pour l'indice de consommation. Par contre, les différences ne sont pas significatives dans la série I.

\section{Série I:}

I'examen détaillé montre que, dans le lot $Y_{2}$, un animal apparemment normal a présenté une croissance nettement aberrante $438 \mathrm{~g}$ par jour), abaissant ainsi sensiblement la moyenne, ce qui explique que la différence obtenue ne soit pas significative. Il est intéressant de noter, à ce propos, que les très fortes variations observées dans la teneur en vitamine $B_{2}$ et $P P$ ne paraissent pas influer sur la vitesse de croissance des animaux observés, ni d'ailleurs sur leur appétit.

La diversité d'origine et de détails opératoires n'a donc pas eu de notable répercussion sur la "valeur alimentaire " des levures, dans les conditions où elles ont été employées.

\section{Série II :}

Par contre, les comparaisons de la série II sont fort intéressantes, car les animaux qui ont réalisé la meilleure croissance (lot $Y_{4}$ ) sont ceux qui ont mangé le moins. Il ne peut, dans ce cas, être invoqué une plus forte appétence de la ration comme explication de sa supériorité.

Rappelons, à ce propos, que nous avons déjà eu l'occasion de comparer deux levures de mélasse produites par la même usine ; les meilleurs résultats avaient été obtenus avec celle qui avait été séchée à haute température, mais ils semblaient alors pouvoir être expliqués par une plus forte consommation, ce qui n'est pas le cas dans la présente série II. I1 paraît en tout cas intéressant de noter que, sur des levures de mélasse tout au moins, le séchage à haute température semble exercer une action favorable. 